\title{
Graphene Oxide Functionalized Double-Layered Patch with Anti-Adhesion Ability for Abdominal Wall Defects
}

\author{
Jian Liu ${ }^{1,2, *}$ \\ Jinfei Hou ${ }^{1,2, *}$ \\ Shaokai Liu (iD) 1,2 \\ Jialun Li iD ${ }^{1,2}$ \\ Muran Zhou ${ }^{1,2}$ \\ Jiaming Sun ${ }^{1,2}$ \\ Rongrong Wang ${ }^{1,2}$ \\ 'Department of Plastic Surgery, Union \\ Hospital, Tongji Medical College, \\ Huazhong University of Science and \\ Technology, Wuhan, 430022, People's \\ Republic of China; ${ }^{2}$ Wuhan Clinical \\ Research Center for Superficial Organ \\ Reconstruction, Wuhan, 430022, People's \\ Republic of China
}

*These authors contributed equally to this work
Background: Effective repair of full-thickness abdominal wall defects requires a patch with sufficient mechanical strength and anti-adhesion characteristics to avoid the formation of hernias and intra-abdominal complications such as intestinal obstruction and fistula. However, patches made from polymers or bio-derived materials may not meet these requirements and lack the bionic characteristics of the abdominal wall.

Materials and Methods: In this study, we report a consecutive electrospun method for preparing a double-layer structured nanofiber membrane (GO-PCL/CS-PCL) using polycaprolactone (PCL), graphene oxide (GO) and chitosan (CS). To expand the bio-functions (angiogenesis/reducing reactive oxygen species) of the patch (GO-PCL/NAC-CS-PCL), $\mathrm{N}$-acetylcysteine (NAC) was loaded for the repair of full-thickness abdominal wall defects $(2 \times 1.5 \mathrm{~cm})$ in rat model.

Results: The double-layered patch (GO-PCL/NAC-CS-PCL) showed excellent mechanical strength and biocompatibility. After 2 months, rats treated with the patch exhibited the desired repair effect with no hernia formation, less adhesion (adhesion score: $1.50 \pm 0.50$, $\mathrm{P}<0.001$ ) and more collagen deposition (percentage of collagen deposition: $34.94 \% \pm 3.31 \%$, $\mathrm{P}<0.001$ ).

Conclusion: The double-layered nanomembranes presented in this study have good antihernia and anti-adhesion effects, as well as improve the microenvironment in vivo. It, therefore, holds good prospects for the repair of abdominal wall defects and provides a promising key as a postoperative anti-adhesion agent.

Keywords: abdominal wall defects, graphene oxide, chitosan, $\mathrm{N}$-acetylcysteine, double layer, electrospinning

\section{Introduction}

Abdominal wall defects caused by trauma, tumor resection, infection, and incisional hernia are common globally, affecting millions of people around the world in recent years. ${ }^{1}$ Traditional synthetic meshes such as polypropylene, and polytetrafluoroethylene are some of the most widely used materials in clinical practice. However, these implants are non-degradable, when they are used alone, adhesions of the abdominal organs may be frequent complications after surgery which further causes intestinal obstruction and fistula. ${ }^{2}$ In order to solve the above problems, biological meshes, including small intestinal submucosa and acellular dermal matrix, have been investigated for use in the repair of abdominal wall defects. The results from these studies that although the biological patches had good
Correspondence: Rongrong Wang; Jiaming Sun

Department of Plastic Surgery, Union Hospital, Tongji Medical College, Huazhong University of Science and Technology, 1277 Jiefang Avenue, Wuhan, 430022, People's Republic of China

Tel +86- I89955 I I I I3;

Tel +86-13986246496

Fax $+86-027-85726240$

Email wangrongrong1988@I26.com;

2004xh080I@hust.edu.cn 
biocompatibility, their mechanical strength was poor, leading to the recurrence of the hernia. ${ }^{3,4}$ Thus, further studies are required to develop better patches.

The abdominal wall consists of the skin, subcutaneous tissues, and the parietal peritoneum. In order to repair defects of the abdominal wall and recover its function, it is necessary to pay attention to the reconstruction of the subcutaneous tissue and parietal peritoneum, which play an important role in resisting intra-abdominal pressure to prevent development of hernia and adhesions, respectively. ${ }^{5}$ Therefore, the use of double-layered patches that can simulate the function of the abdominal wall may be able to achieve a good outcome of abdominal wall repair.

In order to simulate the strong tension provided by the fascia and muscle, polycaprolactone (PCL) and graphene oxide (GO) were used to prepare the first layer of the patch. PCL is a FDA-approved synthetic polymer, which is nontoxic, biocompatible, and biodegradable. ${ }^{6,7}$ Studies by Hympanova et al showed that the use of PCL for the repair of abdominal wall defects was associated with insufficient mechanical strength to prevent the development of hernias. ${ }^{8}$ Furthermore, its poor hydrophilicity results in low cellular affinity, which limits its individual use in biomedical applications. GO is one of the carbon nanomaterials with the highest hardness. ${ }^{9}$ Due to its excellent inherent properties, when it is embedded and uniformly dispersed in other materials, it can increase the tensile strength and Young's modulus of the composite scaffold. ${ }^{10}$ Therefore, mixing GO and PCL to prepare a composite scaffold may solve the problem of insufficient mechanical strength associated with simple PCL scaffolds. In addition, $\mathrm{GO}$ has a lot of hydrophilic functional groups on its surface, which makes it a promising biomaterial for regulating behavior of cells. ${ }^{11}$

To further prevent formation of adhesions, chitosan (CS) was introduced to prepare the second layer of the patch to simulate the function of parietal peritoneum. CS, a natural component of shells or other crustaceans, is biocompatible and biodegradable. ${ }^{12}$ Moreover, it is also the deacetylated derivative of chitin, which is commonly used for preventing adhesions in the clinic. In addition, several studies have shown that CS-based scaffolds have hemostatic and antibacterial capabilities, which can reduce local inflammatory reactions, and thus prevent formation of adhesions. ${ }^{13,14}$ Furthermore, for promoting the repair of abdominal wall defects, N-acetylcysteine (NAC) was loaded to expand the bio-functions of the patch. NAC is approved by the FDA for clinical use as an antioxidant which has carboxyl thiol groups. ${ }^{15}$ In recent years, more and more studies have suggested that NAC has the role of promoting neovascularization, and can reduce the levels of reactive oxygen species (ROS) at the point of injury area. $^{16,17}$ Moreover, in theory, the carboxyl group of NAC can form an amide bond with the amino group of CS under the activation of the EDC/NHS system to achieve the purpose of drug loading. ${ }^{18}$

Based on the above four materials, we used the electrospinning technology to construct a double-layered patch for the repair of abdominal wall defects. Electrospinning technology is a commonly used method in the field of tissue engineering. It permits the production of extracellular-matrix-like three-dimensional structures with nanoscale fibers which have various advantageous characteristics, such as large area-to-volume ratio and high porosity. ${ }^{19} \mathrm{In}$ this study, a blend of GO and PCL was first used to generate the outer scaffold (GO-PCL). It should be noted that the use of different concentration of GO affects the mechanical properties and biocompatibility of the final scaffold. Thus, it was necessary to first determine the optimal concentration of GO that would give a GO-PCL scaffold with high mechanical strength and low cytotoxicity. Thereafter, the inner scaffold (CS-PCL) was prepared by spraying CS and PCL directly onto the surface of the GO-PCL scaffold to form a double-layered scaffold (GOPCL/CS-PCL). Finally, NAC was loaded to the GO-PCL /CS-PCL scaffold to obtain the final drug-loaded scaffold (GO-PCL/NAC-CS-PCL). The diagram showing the experimental design is shown in Figure 1.

\section{Materials and Methods Fabrication of GO-PCL Scaffolds}

PCL (molecular weight 80,000), dissolving agent Dichloromethane (DCM) and Dimethylformamide (DMF) were purchased from Sigma-Aldrich. GO was prepared by using Hummer's Method as described in our previous study. ${ }^{20}$ PCL was dissolved in DCM under continuous magnetic stirring (LC-RE-201D, China) for 1 hour to obtain a solution with PCL concentration of $20.0 \% \mathrm{w} / \mathrm{v}$. GO $(0.4 \mathrm{~g})$ was dissolved in $8 \mathrm{~mL}$ DMF using ultrasonication (Branson, USA) in an ice-bath for 2 hours to obtain homogeneous GO/DMF dispersion. Thereafter, different volumes of the GO/DMF dispersion were added to the PCL/DCM solution, and stirred for 30 minutes to 


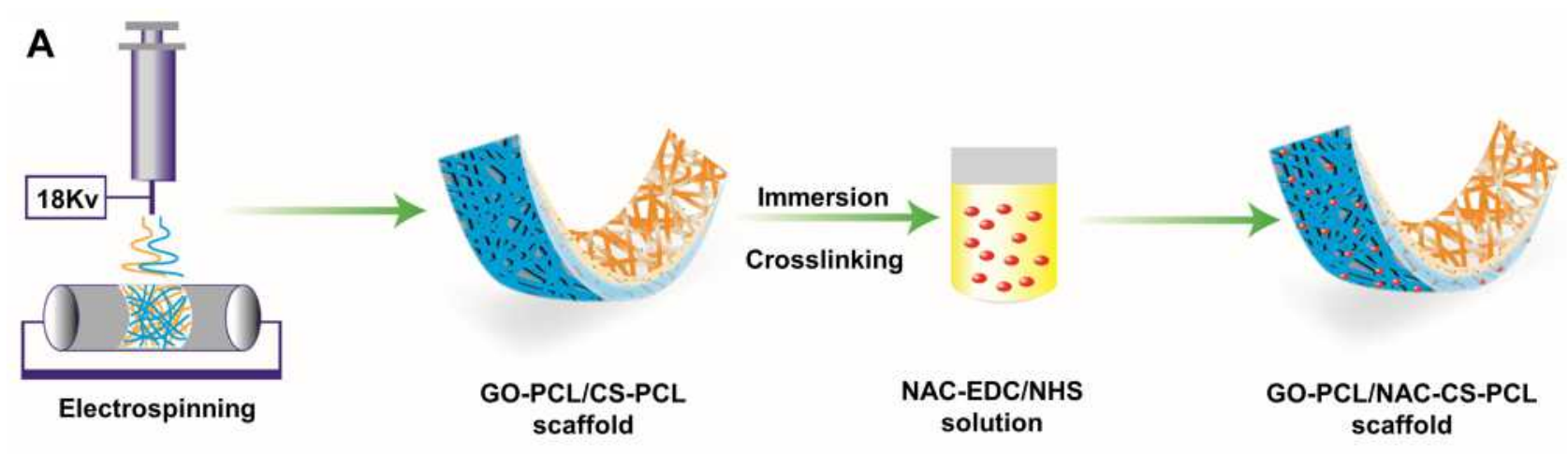

B

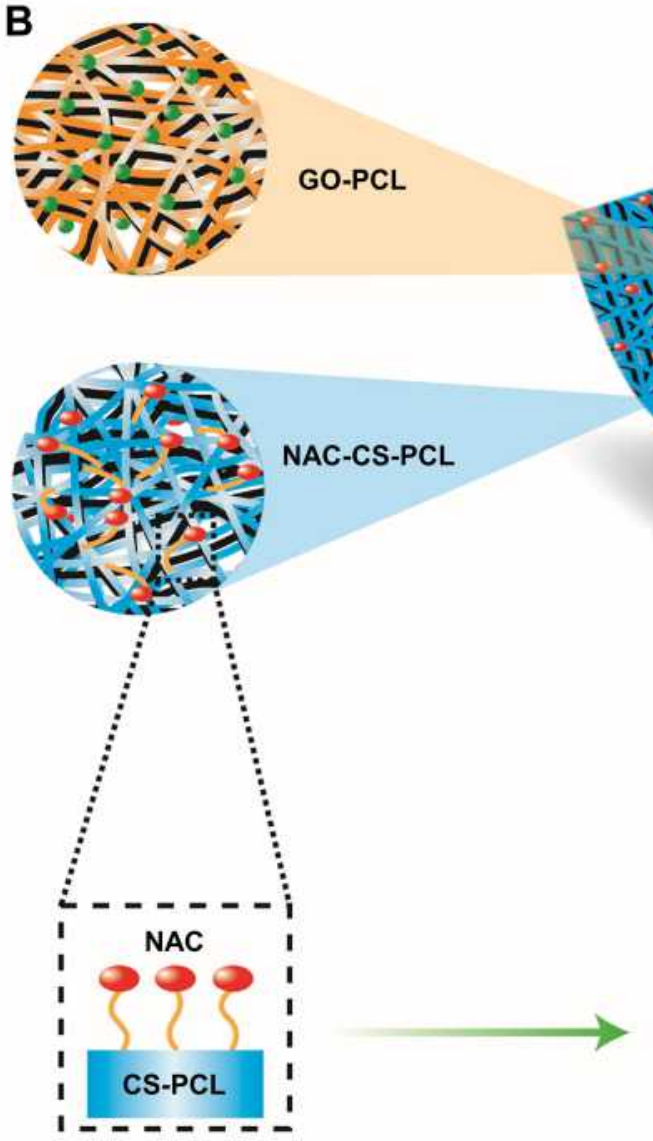

GO-PCL/NAC-CS-PCL

scaffold

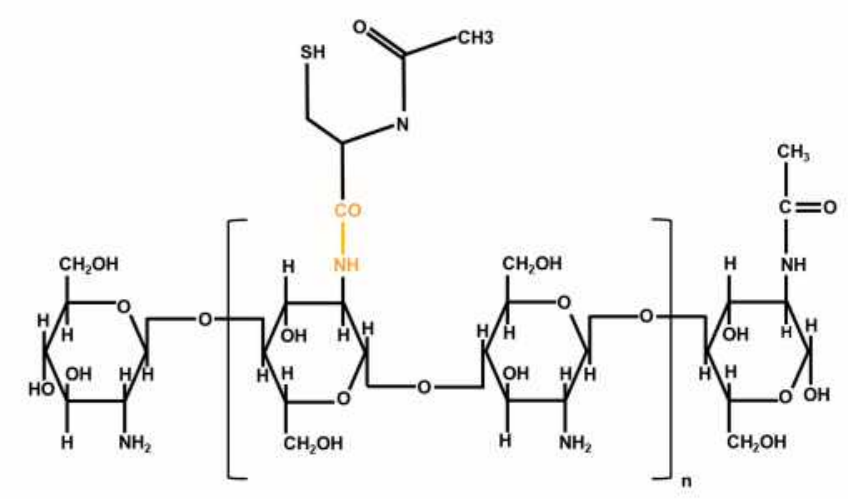

Graphene oxide (GO)

$\mathrm{N}$-acetylcysteine (NAC)

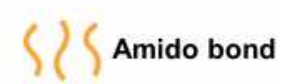

GO-PCL

CS-PCL

Figure I Experimental design.

Notes: (A) An illustration of the design and fabrication process of the scaffold. A blend of GO and PCL was firstly electrospun to fabricate outer scaffold (GO-PCL). Next, the inner scaffold (CS-PCL) was sprayed directly on the surface of GO-PCL scaffold to obtain a double-layer scaffold (GO-PCL/CS-PCL). The prepared GO-PCL/CS-PCL scaffold was immersed in NAC-EDC/NHS solution and crosslinked to obtain a final scaffold (GO-PCL/NAC-CS-PCL). (B) Illustration of GO-PCL/NAC-CS-PCL scaffold and its application in the reconstruction of full-thickness abdominal wall defect $(2.0 \mathrm{~cm} \times 1.5 \mathrm{~cm})$ in rats.

obtain a series of GO-PCL compound with GO concentrations of $0.0,0.1,0.5$, and $1.0 \% \mathrm{w} / \mathrm{v}$.

For the electrospinning process, the mixture was filled in a $10-\mathrm{mL}$ syringe with a 19-gauge needle. Electrospinning was then performed for 12 hours to generate nanofiber mats with enough thickness using the following parameters: supplied voltage, $18 \mathrm{kV}$; needle tip to collector distance, $10 \mathrm{~cm}$; flow rate, $1 \mathrm{~mL} / \mathrm{h}^{21}$ The nanofiber scaffolds (including PCL, 0.1\%GO-PCL, 0.5\% GO-PCL, $1.0 \% \mathrm{GO}-\mathrm{PCL})$ that were generated were 
subsequently dried overnight in a vacuum oven to remove residual solvents. In order to screen out the scaffolds with sufficient mechanical strength and good biocompatibility for subsequent experiments, we used PCL scaffolds as the control group, and 0.1\%GO-PCL, 0.5\%GO-PCL, $1.0 \%$ GO-PCL scaffolds as the experimental group. And the physiochemical properties and biocompatibility were assessed in vitro.

\section{Physiochemical Characterization of GO-PCL Scaffolds}

\section{Morphology of GO-PCL Scaffolds}

The microstructure of the nanofibers was observed using a Field Emission Scanning Electron Microscope (FESEM, Nova). Before observation, all the samples were coated with gold for $60 \mathrm{~s}$ using a sputter coater. ${ }^{22}$ The diameter of the generated nanofibers was determined by calculating the average diameter from nine regions of the nanofibers (10 points per region) in representative images using Adobe Illustrator CS6 Software.

\section{Raman Spectra Analysis}

The functional groups present in the pure GO, and GOPCL composite nanofibers were examined using a Dispersive Raman Microscope (FRA 106/s, Bruker, Germany) in the range of $0-4000 \mathrm{~cm}^{-1}$ with a wavelength of $532 \mathrm{~nm}$ at room temperature.

\section{Mechanical Property Testing}

Before examination, all the samples were sectioned into rectangular shapes with a test dimension of $15 \times 5 \mathrm{~mm}$. The mechanical properties of the GO-PCL scaffolds were determined by using all-electric dynamic test instrument (Instron, British) at a speed of $5 \mathrm{~mm} / \mathrm{min}$. Four samples were tested for each type of the GO-PCL nanofiber scaffolds, and the Young's modulus were calculated using the OriginPro 8 Software.

\section{In vitro Cytocompatibility of GO-PCL Scaffolds \\ Cell Culture and Seeding on Scaffolds}

Human dermal fibroblasts (HDFs, ATCC, America) were cultured in complete Dulbecco's Modified Eagle's Medium (DMEM, Thermo Fisher Scientific, America) supplemented with $10 \%$ fetal bovine serum and $1 \%$ penicillin/streptomycin at $37^{\circ} \mathrm{C}$ and $5 \% \mathrm{CO}_{2}$. When the cells reached $80 \%$ confluency, they were washed with phosphate-buffered saline and trypsinized with $0.25 \%$ Trypsin-
EDTA for passaging and seeding. The simple PCL and GO-PCL scaffolds were cut into square shapes with a length of $0.5 \mathrm{~cm}$, and sterilized with ethylene oxide at $37^{\circ} \mathrm{C}$ overnight. Then, the sterilized scaffolds were fitted onto the bottom of a 96-well tissue culture plate and washed with PBS three times followed by incubating in completed DMEM for 4 hours. The HDFs were then seeded into the 96-well culture plates at a density of $3 \times 10^{3}$ cells per well.

\section{Cell Viability and Proliferation}

After 1, 4 and 7 days of culture, cell proliferation on the simple PCL and GO-PCL scaffolds was assessed using the Cell Counting Kit-8 (CCK-8, Sigma-Aldrich) assay according to the manufacturer's protocol. In brief, 200ul CCK-8 working solution was added to each well and incubated at $37^{\circ} \mathrm{C}$ for 1 hour. Afterwards, 100ul supernatant solution was transferred to a new 96-well plate, and the optical density (OD) of the solution was examined using a microplate reader at $450 \mathrm{~nm}$.

\section{Fabrication of GO-PCL/NAC-CS-PCL Composite Nanofiber Scaffold}

After comprehensive analysis of the above test results, we selected a GO-PCL scaffold with optimal GO concentration for further analysis. CS (molecular weight 50,000190,000; 95\% deacetylated), dissolving agent 1,1,1,3,3,3-hexafluoro-2-propanol (HFIP) and 2,2,2-trifluoroethanol (TFE) were purchased from Sigma-Aldrich. CS was added to a mixture of HFIP and TFE (HFIP: $\mathrm{TFE}=60: 40 \mathrm{v} / \mathrm{v} \%$ ), and dissolved in an oil bath at $50^{\circ} \mathrm{C}$ for 24 hours using a magnetic stirrer (LC-RE-201D, China). Thereafter, PCL (PCL:CS=90:10 w/w\%) was added to the chitosan solution, and stirred for 24 hours at room temperature to obtain a solution with a total concentration of $5.0 \% \mathrm{w} / \mathrm{v}$.

For the electrospinning process, the GO-PCL scaffolds with optimal GO concentration $(0.1 \% \mathrm{w} / \mathrm{v})$ acted as the outer scaffold and were prepared as described in the preceding sections. Subsequently, the inner scaffolds were prepared by electrospinning the CS-PCL solution directly onto the surface of the GO-PCL scaffolds for another 6 hours using the same parameters. In this way, doublelayered GO-PCL/CS-PCL scaffolds were obtained. After drying overnight in a vacuum oven, the GO-PCL/CS-PCL scaffolds were immersed in a mixture of $50 \mathrm{mM}$ EDC (Sigma-Aldrich) and $12.5 \mathrm{mM}$ NHS (Sigma-Aldrich). NAC was loaded onto the scaffolds by adding $0.5 \mathrm{mg} / \mathrm{mL}$ 
NAC (Sigma-Aldrich) into the EDC/NHS system. After soaking for 24 hours, the drug-loaded scaffolds (GO-PCL /NAC-CS-PCL) were obtained. In this part of the experiment, we used PCL, 0.1\%GO-PCL, 0.1\%GO-PCL/CSPCL scaffolds as the control group, and $0.1 \% \mathrm{GO}-\mathrm{PCL}$ /NAC-CS-PCL scaffolds as the experimental group, and further explored the physiochemical properties, biocompatibility, and repair effect of abdominal wall defect of the $0.1 \%$ GO-PCL/NAC-CS-PCL scaffolds.

\section{Physiochemical Characterization and in vitro Biocompatibility of the GO-PCL/ NAC-CS-PCL Scaffolds}

Morphology, Raman Spectra and Mechanical Property of the Scaffolds

The microstructure of the GO-PCL/NAC-CS-PCL scaffolds was observed using FESEM as described in the preceding sections, while the functional groups of NAC present in the GO-PCL/NAC-CS-PCL scaffolds were examined using Dispersive Raman Microscope. In addition, the mechanical properties for each type of scaffold were tested in four samples.

\section{Cell Viability, Proliferation and Cell Adhesion, Morphology}

LIVE/DEAD assay (Sigma-Aldrich), and CCK-8 assay were conducted to compare the cell viability and proliferation ability on the four types of scaffolds, which included simple PCL, 0.1\%GO-PCL, 0.1\%GO-PCL/CS-PCL and $0.1 \%$ GO-PCL/NAC-CS-PCL scaffolds. Live and dead cells were stained with fluorescein diacetate/propidium iodide (FDA/PI) (Sigma-Aldrich), respectively, and observed using a laser scanning confocal microscopy (LSCM, Nikon A1Si, Japan). Specifically, the greenstained cells were living cells, and red-stained cells were dead. Cell adhesion and morphology were observed using FESEM. Typically, 1 day after incubation, HDFs on the $0.1 \%$ GO-PCL/NAC-CS-PCL scaffolds were washed three times with PBS to remove unattached cells, and fixed with $2.5 \%$ glutaraldehyde for 2 hours. Then, they were washed again with PBS to remove residual glutaraldehyde, and dehydrated with increasing concentrations of ethanol (50\% for $60 \mathrm{~min}, 70 \%$ for $30 \mathrm{~min}, 80 \%$ for $20 \mathrm{~min}, 90 \%$ for $10 \mathrm{~min}, 95 \%$ for $5 \mathrm{~min}, 100 \%$ for $5 \mathrm{~min}$ ). Finally, all the samples were dried in air, and coated with platinum for $60 \mathrm{~s}$ in a vacuum and observed using FESEM.

\section{In vitro NAC Release}

Four samples of $0.1 \% \mathrm{GO}-\mathrm{PCL} / \mathrm{NAC}-\mathrm{CS}-\mathrm{PCL}$ scaffold were immersed in $2 \mathrm{~mL}$ of PBS at $37^{\circ} \mathrm{C}$. At 1, 2, 4, 8, $24 \mathrm{~h}$, and 2, 7, 14 days, the soaking solution was filtered to remove the residue and transferred to Eppendorf tubes. The released NAC concentration was measured using high-performance liquid chromatography (HPLC) as previously described. ${ }^{18}$

\section{In vivo Study on Repair of Abdominal Wall Defects Surgery Procedures}

Four groups $(n=6)$ of male Sprague-Dawley rats with initial average weight of $220-250 \mathrm{~g}$ were used as hosts. All the animal treatments and procedures were implemented according to the ethical guidelines of Tongji Medical College, Huazhong University of Science and Technology (NO. 2019-S069). After being anesthetized with intraperitoneal injection of $3 \%$ pentobarbital sodium $(0.1 \mathrm{~mL}$ per $100 \mathrm{~g}$ ), a $2.0 \mathrm{~cm} \times 1.5 \mathrm{~cm}$ full size thickness abdominal wall defect was created in the middle ventral abdominal wall. Thereafter, PCL, 0.1\%GO-PCL, 0.1\%GO-PCL/CS-PCL, $0.1 \% \mathrm{GO}-\mathrm{PCL} / \mathrm{NAC}-\mathrm{CS}-\mathrm{PCL}$ scaffolds of $3.0 \mathrm{~cm} \times 2.0 \mathrm{~cm}$ were applied to repair the defect in the four groups, respectively. Scaffolds were implanted using 6-0 Prolene sutures in a continuous suture pattern. Skin incisions were closed with 3-0 Prolene sutures in a continuous suture pattern combined with interrupted sutures. After recovery, all the rats had free access to food and water, and the number of hernias formed and deaths in each group were recorded. Two months after implantation, the rats were sacrificed using an overdose of anesthetics, and the presence of adhesions was evaluated by two investigators who were blind to the categorized groups. The abdominal wall which included repaired tissues and residual scaffold was removed en bloc for histology and immunohistochemistry analysis.

\section{Adhesion Evaluation}

For evaluation of adhesions, a U-shaped incision was made carefully in the abdominal wall without destroying any adhesions. Adhesion score was evaluated according to the modified scale devised by Nair et al combined with the criteria described by Walker: 0 , no adhesions; 1 , single band of adhesions between the viscera or from viscous to the abdominal wall; 2, two bands, either between viscera or from viscera to the abdominal wall, or three bands but easily freed by blunt dissection 3 , more than three bands 
between viscera or from viscera to the abdominal wall; 4, viscera directly adherent to the abdominal wall, irrespective of the number and extent of adhesive bands. ${ }^{23}$

\section{Histology and Immunohistochemistry Staining}

All the tissue samples were fixed with $4 \%$ paraformaldehyde, paraffin-embedded, and cut into 5 um sections containing the biomaterials and the surrounding native tissue. For the evaluation of granulation and new tissues, deparaffinized sections were first stained with hematoxylin and eosin (HE). In order to assess collagen deposition, we performed Masson's Trichrome stain and Sirius Red stain, and further observed the results of Sirius Red staining under a polarized light microscope to distinguish the type of collagen. Neovascularization were evaluated using immunehistochemical staining of CD31. The mean number of new vessels was calculated at five randomly selected high magnification $(\times 400)$ fields using Image-Pro Plus.

\section{Statistical Analysis}

All experimental results were expressed as mean \pm standard deviation and analyzed using Tukey's $t$-test and one-way ANOVA by GraphPad Prism 8 Software. The level of significance was noted by $* * *$ and $* * *$ which refer to the $\mathrm{P}$ value of $<0.05,<0.01$, and $<0.001$, respectively.

\section{Results}

\section{Morphology of the GO-PCL Scaffolds}

Compared to PCL scaffolds, as shown in Figure 2, FESEM was used to evaluate the morphology of GO-PCL scaffolds at different concentrations of GO $(0.1,0.5$, and $1.0 \% \mathrm{w} / \mathrm{v})$ and the fiber diameters for each sample were measured. Each sample was shown with one macrograph, one lower magnification and one higher magnification image and graphical representation of fiber frequency range. We found that most of these nanofibers were distributed between 250 and $350 \mathrm{~nm}$ in diameter, while the structure was randomly oriented to form a three-dimensional porous structure. However, at $1.0 \%$ GO-PCL, some nanofibers were broken, which were indicated with the red arrow in Figure 2A. The results showed that we had successfully prepared GO-PCL scaffolds with different concentrations of GO by electrospinning technology.

\section{Physiochemical Properties of the GO-PCL Scaffolds}

Raman spectrum was used to analyze the carbon components in pure GO powder and GO-PCL scaffolds. As shown in Figure 2B, characteristic peak $\mathrm{D}$ band $\left(1323 \mathrm{~cm}^{-1}\right)$ and $\mathrm{G}$ band $\left(1583 \mathrm{~cm}^{-1}\right)$ were observed in the GO powder. After GO was incorporated into PCL, the characteristic D and $\mathrm{G}$ bands peaks were also observed, which indicated that GO had been successfully introduced into the GO-PCL scaffolds. The tensile stress-strain curves of the different GO-PCL scaffolds are shown in Figure 2C. The mechanical strength and Young's modulus can be evaluated intuitively using the highest point of the ordinate and the line slope of the linear part of the curves, respectively. ${ }^{24}$ Figure $2 \mathrm{C}$ shows that the addition of GO significantly improves the tensile strength of the GO-PCL scaffolds compared with the PCL scaffolds. Among them, $0.5 \% \mathrm{GO}-\mathrm{PCL}$ scaffold had the best tensile strength, while the tensile strength for $0.1 \% \mathrm{GO}-\mathrm{PCL}$ was relatively poor. But there is no statistical difference in the Young's modulus of the different GO-PCL scaffolds, and it is two times higher than that of the simple PCL scaffolds as shown in Figure 2D. As a kind of nanofiller, GO could well disperse in the PCL polymer matrix, and be beneficial to stress transfer from matrix to GO sheet, leading to the improvement of tensile strength. And as the GO concentration increases, the effect will be more obvious. However, when the addition of GO is beyond a certain limit, the GO tends to aggregate, resulting in the reduction of elasticity of the GO-PCL scaffolds. ${ }^{25}$ And this may explain the reason for the result shown in Figure 2C.

\section{Cell Viability and Proliferation on GO-PCL Scaffolds}

Cell viability and proliferation on different GO-PCL scaffolds were analyzed using the CCK- 8 assay. As shown in Figure 2E, the HDFs grew well on the GO-PCL scaffolds with the extended culture time. However, as the concentration of GO in the scaffold increased, the rate of cell proliferation rate decreased, particularly for the scaffolds with $0.5 \%$ and $1.0 \%$ GO. Therefore, we chose the $0.1 \%$ GO-PCL scaffold for further experiment.

\section{Morphology of the $0.1 \% \mathrm{GO}-\mathrm{PCL} / \mathrm{NAC}$ - CS-PCL Scaffolds}

After comprehensive analysis of the physiochemical properties and cytocompatibility of different GO-PCL scaffolds, we selected the $0.1 \% \mathrm{GO}-\mathrm{PCL}$ scaffold and constructed $0.1 \% \mathrm{GO}-\mathrm{PCL} / \mathrm{CS}-\mathrm{PCL}$ and $0.1 \% \mathrm{GO}-\mathrm{PCL}$ NAC-CS-PCL scaffolds for further analysis. As shown in Figure $3 \mathrm{~A}$, the macrograph, surface topography and 


\section{A \\ 음}

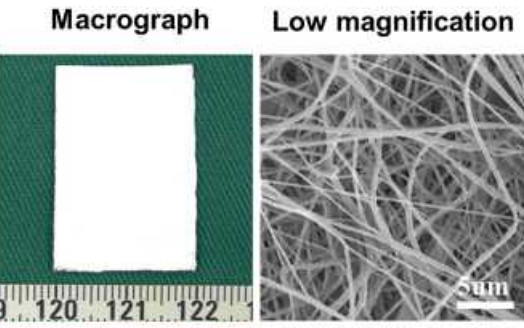

High magnification

Nanofiber diameter
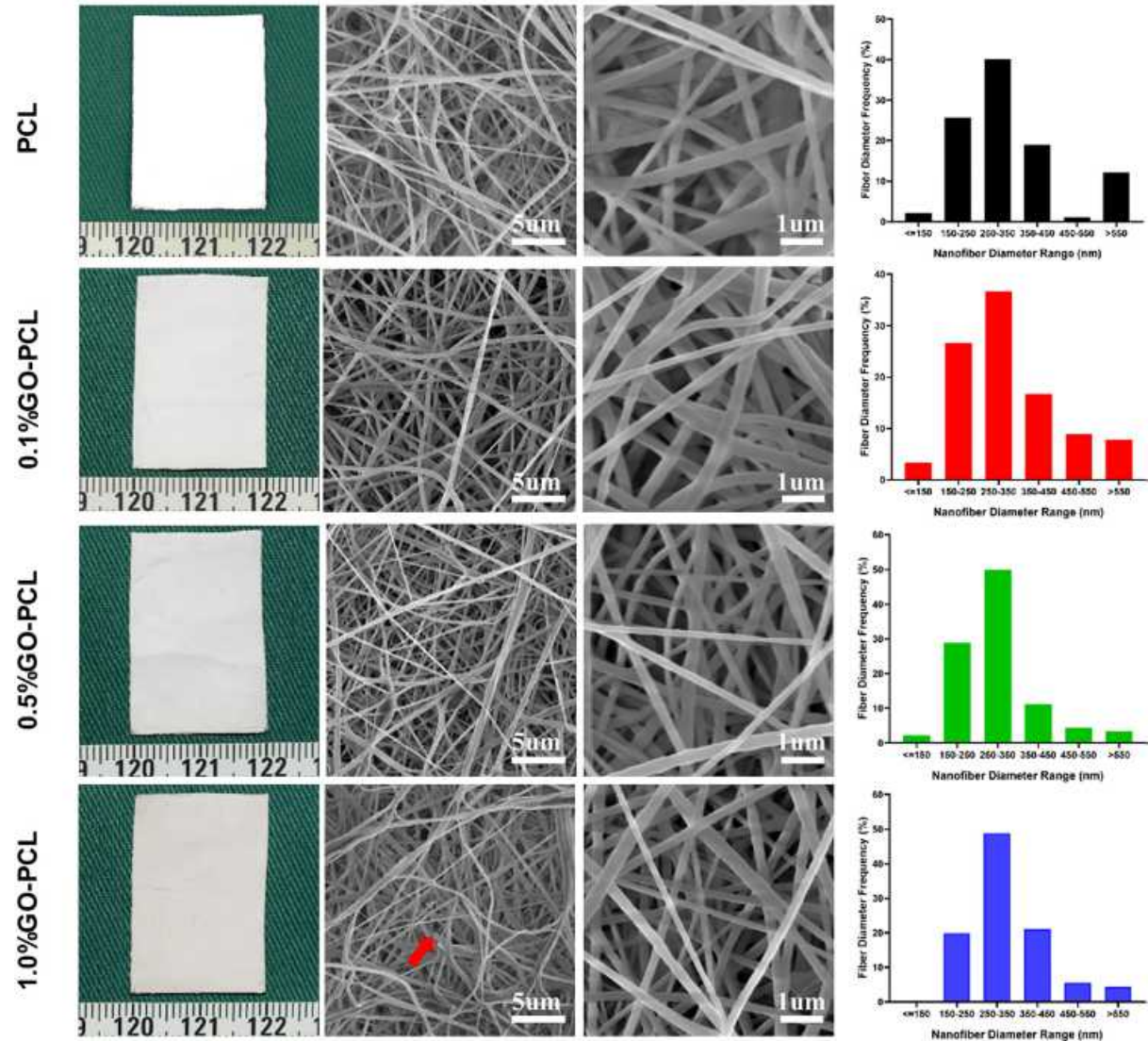

B
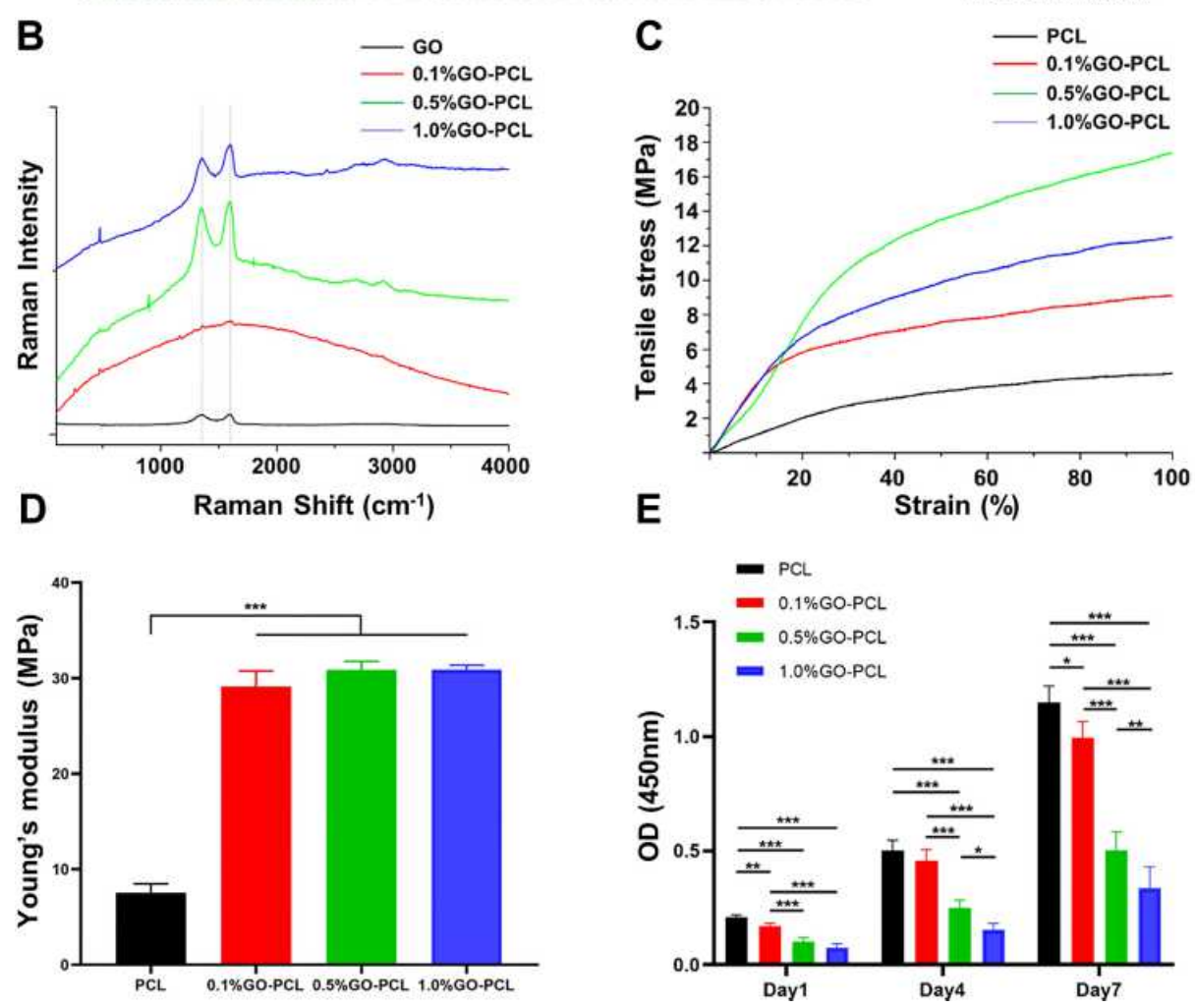

Figure 2 Characterization of the GO-PCL scaffolds.

Notes: (A) Macrographs, FESEM images, and histogram showing the nanofiber diameter of GO-PCL scaffolds with different concentrations of GO. The red arrow indicates the broken nanofiber; (B) Raman spectroscopy, (C) tensile stress-strain curve and (D) Young's modulus of the scaffolds; (E) cell proliferation of HDFs seeded onto the scaffolds was assessed using the CCK-8 assay (mean $\pm \mathrm{SD} ; * \mathrm{P}<0.05, * * \mathrm{P}<0.0 \mathrm{I}$, *** $\mathrm{P}<0.00 \mathrm{I}$ ). 
cross-section view under SEM of these two scaffolds were similar and they both had a double-layered structure.

\section{Physiochemical Properties of the $0.1 \%$ GO-PCL/NAC-CS-PCL Scaffold}

From the Raman spectroscopy analysis (Figure 3B), the $0.1 \%$ GO-PCL/NAC-CS-PCL scaffolds had the characteristic peak of NAC at $2934 \mathrm{~cm}^{-1}$, which was indication that NAC had been successfully loaded onto the scaffold. Figure $3 \mathrm{C}$ and $\mathrm{D}$ show that the mechanical strength and Young's modulus of the $0.1 \% \mathrm{GO}-\mathrm{PCL} / \mathrm{NAC}-\mathrm{CS}-\mathrm{PCL}$ scaffolds were similar to those of the $0.1 \% \mathrm{GO}-\mathrm{PCL} / \mathrm{CS}$ PCL scaffold, which were significantly higher than the simple PCL scaffolds (Figure 2D).

\section{In vitro NAC Release}

Figure $3 \mathrm{E}$ shows that the drug release curve of the $0.1 \%$ GO-PCL/NAC-CS-PCL scaffold can be divided into three stages. The first stage $(0-4 \mathrm{~h})$ is characterized by relatively rapid release of NAC which can be maintained at a higher concentration in a short time. In the second stage (4h-7d), the curve shows a slow-rising upward trend that eventually flattens gradually. The amount of NAC released at this stage is small. In the third phase $(7 \mathrm{~d}-14 \mathrm{~d})$, the curve enters into the plateau phase, and almost no NAC is released.

\section{Biocompatibility of $0.1 \%$ GO-PCL/NAC- CS-PCL Scaffolds in vitro}

Staining of live and dead cells after 1, 4, and 7 days of culture on different scaffolds is shown in Figure 4A. Compared to the $0.1 \%$ GO-PCL scaffold, fibroblasts had better cell viability as well as higher proliferation rates on other scaffolds. These findings were consistent with the result of the CCK-8 assay (Figure 4B). Specifically, the $0.1 \%$ GO-PCL/NAC-CS-PCL and simple PCL scaffolds had similar cell viability and proliferation rates compared to the $0.1 \%$ GO-PCL/CS-PCL scaffolds. The presence of $\mathrm{NAC}$ induced cell proliferation slightly. Figure 4C shows the morphology of fibroblasts attached to the $0.1 \% \mathrm{GO}-$ PCL/NAC-CS-PCL scaffolds after 1 day of culture as observed using FESEM. The image indicates that the cells successfully adhered to the scaffold and exhibited typical fibroblast-like morphology. The pseudopods derived from mature cells are indicated by red arrows. In summary, it can be shown that the $0.1 \%$ GO-PCL/NAC-CS -PCL scaffolds have good in vitro biocompatibility.

\section{In vivo Abdominal Wall Defects Repair in Rat}

After 2 months of implantation, the rats repaired with different scaffolds (control group: PCL, 0.1\%GO-PCL, $0.1 \% \mathrm{GO}-\mathrm{PCL} / \mathrm{CS}-\mathrm{PCL}$; experimental group: $0.1 \% \mathrm{GO}-$ PCL/NAC-CS-PCL) survived well, but the use of PCL scaffolds was associated with different degrees of hernia formation. However, in the scaffolds composed of GO (0.1\%GO-PCL， 0.1\%GO-PCL/CS-PCL， 0.1\%GO-PCL /NAC-CS-PCL), no hernia was observed. The side and top views of the rats after surgery in the four groups are shown in Figure 5A-D, E-H, respectively. The red dotted round frame indicates the hernias formed in the PCL scaffolds as shown in Figure 5A and E. From Figure $5 \mathrm{E}-\mathrm{H}$, we see that the scaffolds were integrated into the host tissues and a new thin layer of tissue formed on the surface. Moreover, in the $0.1 \% \mathrm{GO}-\mathrm{PCL}$ NAC-CS-PCL group, the new tissue on the surface of the scaffold seemed to be denser as shown in Figure $5 \mathrm{H}$. The results of adhesion evaluation after implantation of the four scaffolds are shown in Figure 5I-L. Compared to the PCL and $0.1 \% \mathrm{GO}-\mathrm{PCL}$ scaffolds, the scaffolds with CS $(0.1 \%$ GO-PCL/CS-PCL，0.1\%GO-PCL/NACCS-PCL) had few to no adhesions, which were easily separated. The histogram showing the adhesion scores of the different scaffolds is shown in Figure 5M.

\section{Histology and Immunohistochemistry Analyses}

To evaluate cell infiltration, collagen deposition, and vessel formation, the rats were sacrificed at 2 months after implantation for HE, Masson's Trichrome, Sirius red staining, and CD31 staining. A panoramic view of the HE staining for the repair of the abdominal defects is shown in Figure 6A-D. In the figures, uppercase letter $\mathrm{C}$ denotes the central area of the reconstruction, which includes residual scaffolds and a small amount of deposited collagen. On the other hand, uppercase letter $J$ represents the junctional area of the reconstruction, which includes a large number of infiltrated cells on the surface of the scaffolds and deposited collagen. High magnification images of the central area (within the red dotted line) are shown in Figure 6E-H. In the groups repaired with PCL, $0.1 \% \mathrm{GO}-$ PCL, and $0.1 \%$ GO-PCL/CS-PCL, only the residual scaffold materials can be observed as indicated using yellow arrows, while cell infiltration cannot be seen. However, in the group repaired with $0.1 \% \mathrm{GO}-\mathrm{PCL} / \mathrm{NAC}-\mathrm{CS}-\mathrm{PCL}$, 

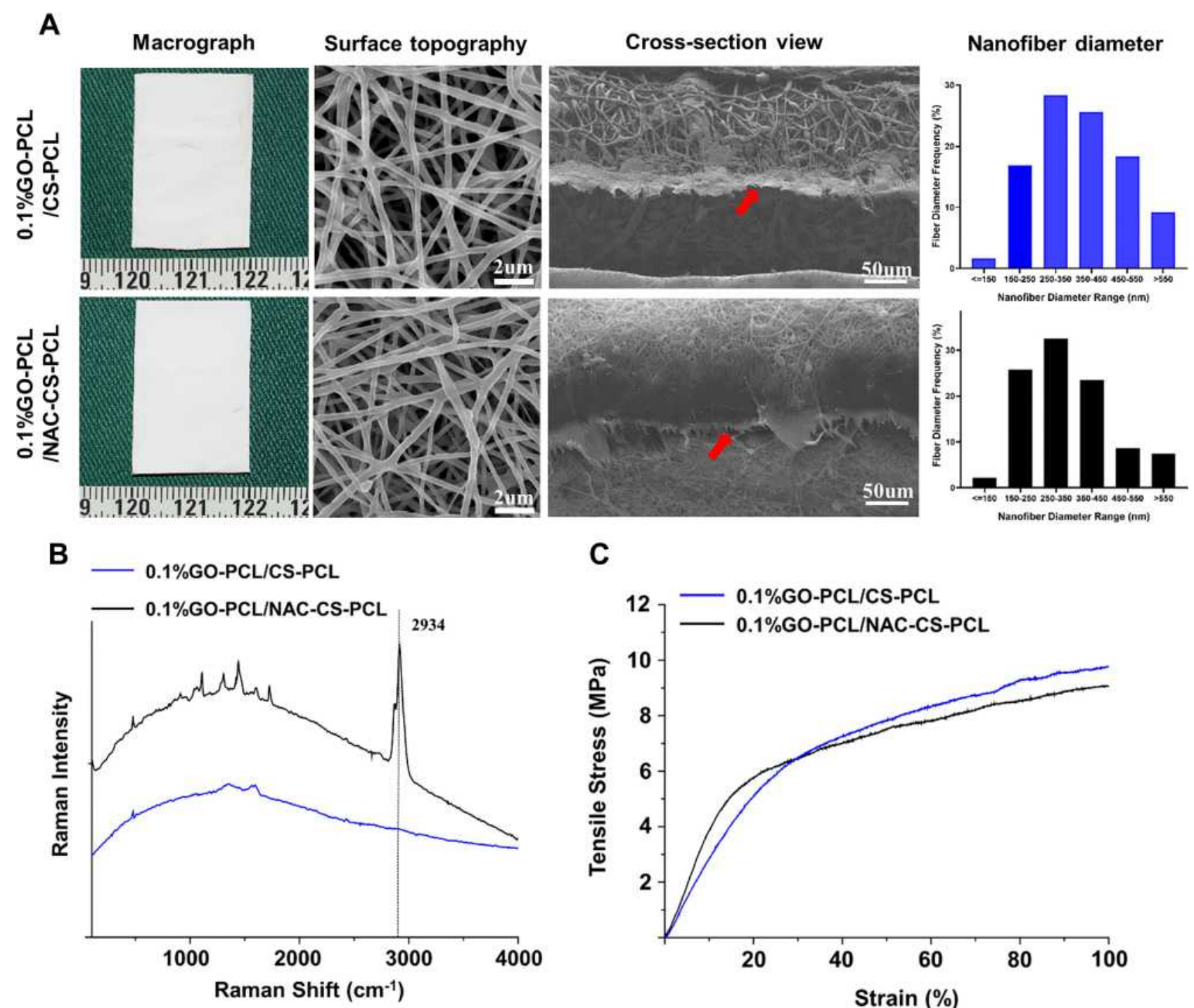

D
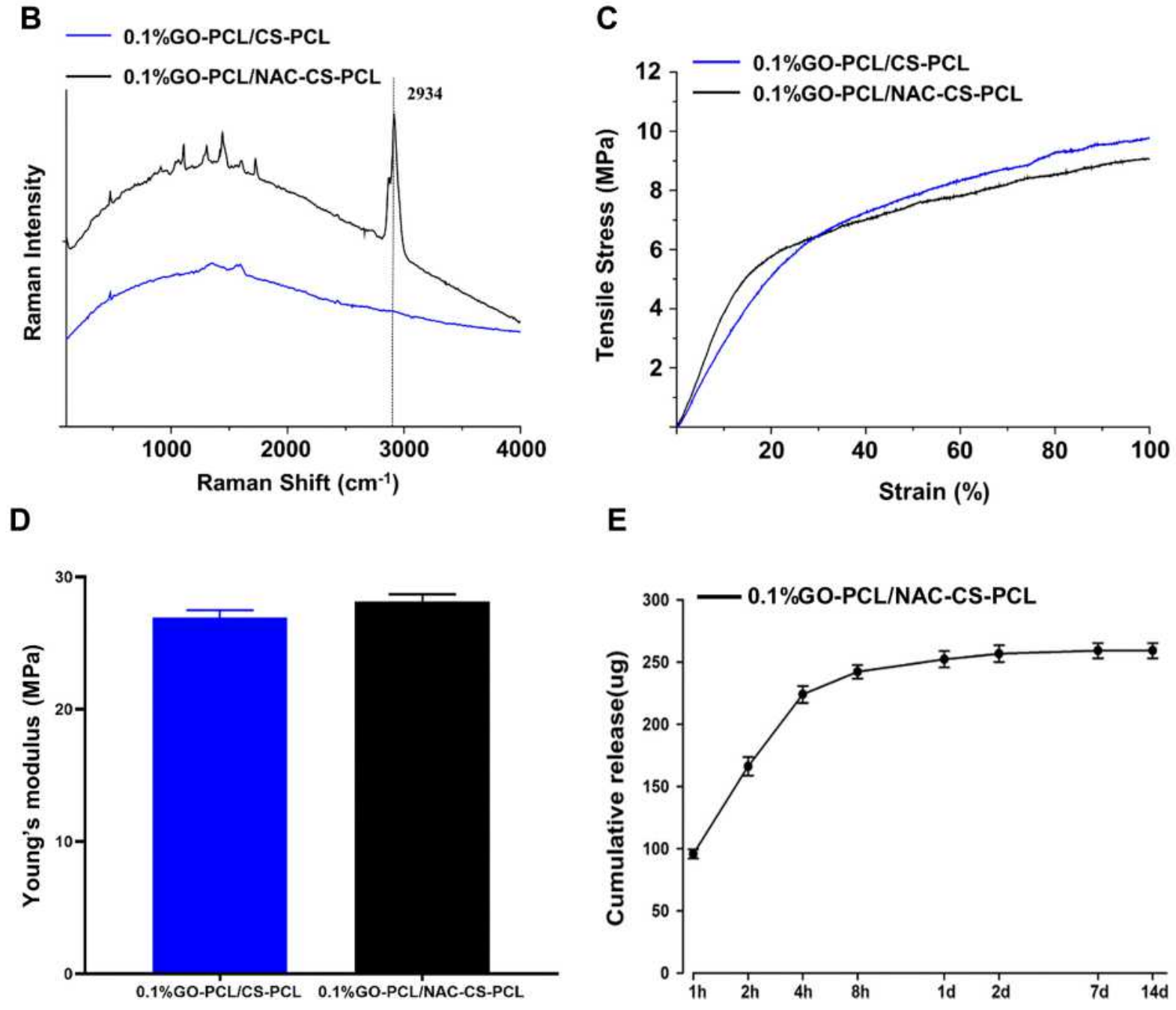

Figure 3 Characterization of the GO-PCL/CS-PCL, GO-PCL/NAC-CS-PCL scaffolds.

Notes: (A) Macrographs, FESEM images, and histogram showing the nanofiber diameter of scaffolds. The red arrow shows the double-layer structure. (B) Raman spectroscopy, (C) tensile stress-strain curve and (D) Young's modulus of the scaffolds. (E) Time-dependent cumulative release profile of NAC from the scaffolds. 


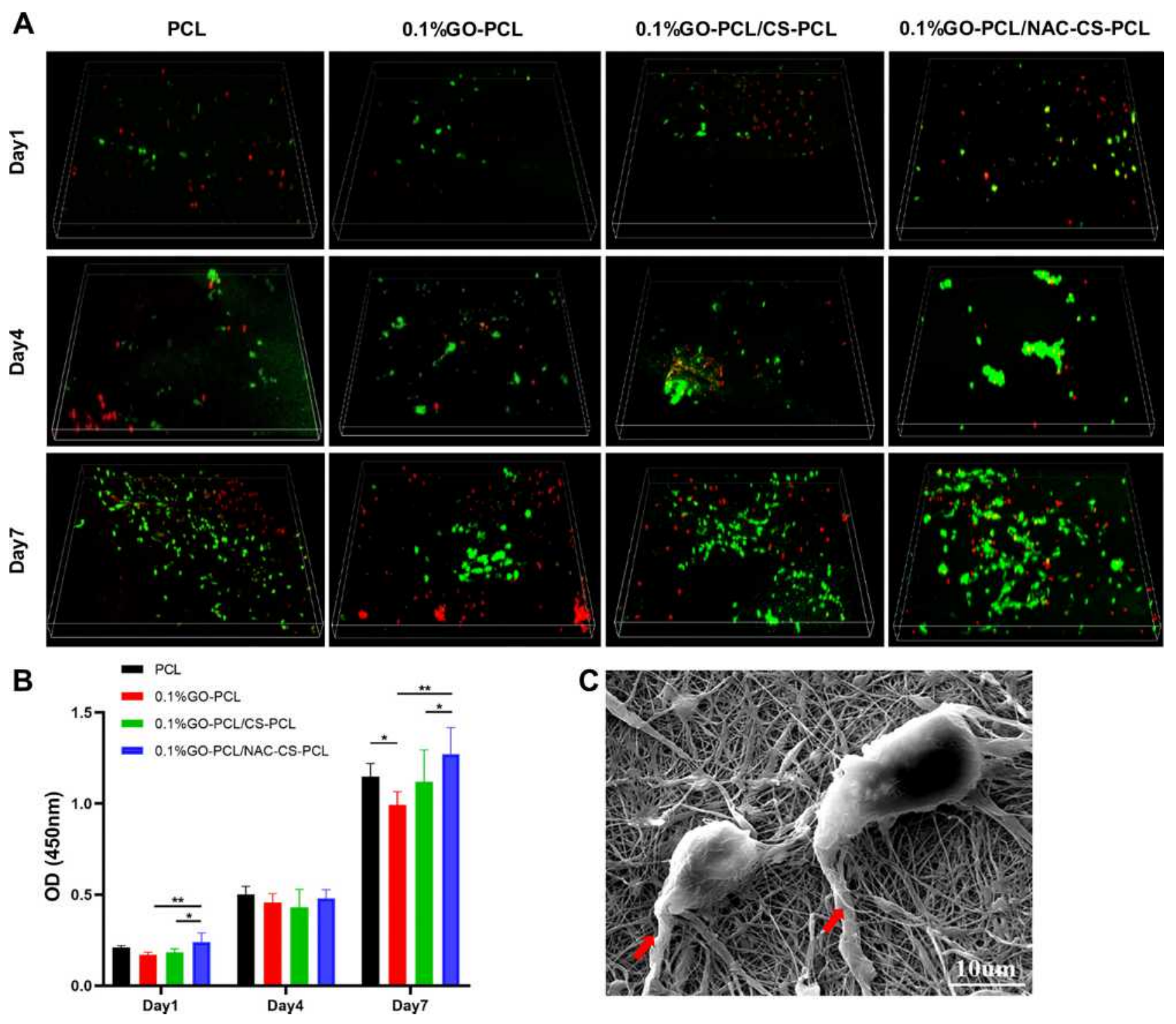

Figure 4 In vitro biocompatibility of the scaffolds.

Notes: (A) Confocal fluorescent images showing results of FDA/PI staining of human dermal fibroblasts cultured on the scaffolds for I, 4 and 7 days. (B) Results of CCK-8 assay performed on human dermal fibroblasts seeded on the scaffolds (mean \pm SD; $* P<0.05, * * P<0.0 I$ ). (C) FESEM image displaying the morphology of human dermal fibroblasts seeded on the $0.1 \%$ GO-PCL/NAC-CS-PCL scaffolds after I day of culture. The red arrow indicates the pseudopods derived from cells.

infiltrated cells as well as new blood vessels (indicated by black arrow) can be seen in the central area. High magnification images of the junctional areas are shown in Figure 6I-L. As can be seen from the images, there were a large number of infiltrating cells and a small amount of residual scaffold material in the junctional area of all the different groups. However, it is worth noting that compared to the PCL group, the infiltrating cells in the other groups were more orderly.

CD31 immunohistochemical staining is shown in Figure $6 \mathrm{M}-\mathrm{T}$. In the central area of reconstruction, neovascularization was only observed in the $0.1 \% \mathrm{GO}-\mathrm{PCL}$ NAC-CS-PCL group but not in the other groups as shown in Figure 6M-P. On the other hand, neovascularization was observed in the junctional areas of all the groups as shown in Figure 6Q-T. Specifically, the $0.1 \%$ GO-PCL/NAC-CS-PCL group had the most number of new blood vessels, while the PCL group had the least. However, there was no statistical difference $(\mathrm{P}=0.32)$ in the number of new blood vessels between the $0.1 \% \mathrm{GO}-$ PCL/NAC-CS-PCL and $0.1 \%$ GO-PCL/CS-PCL group as shown in Figure 6U.

Masson's Trichrome staining is shown in Figure 7A-H. In the central area, except for the $0.1 \%$ GO-PCL/NAC-CSPCL group, only a small amount of collagen was deposited in other three groups, which are indicated by the red arrow. 


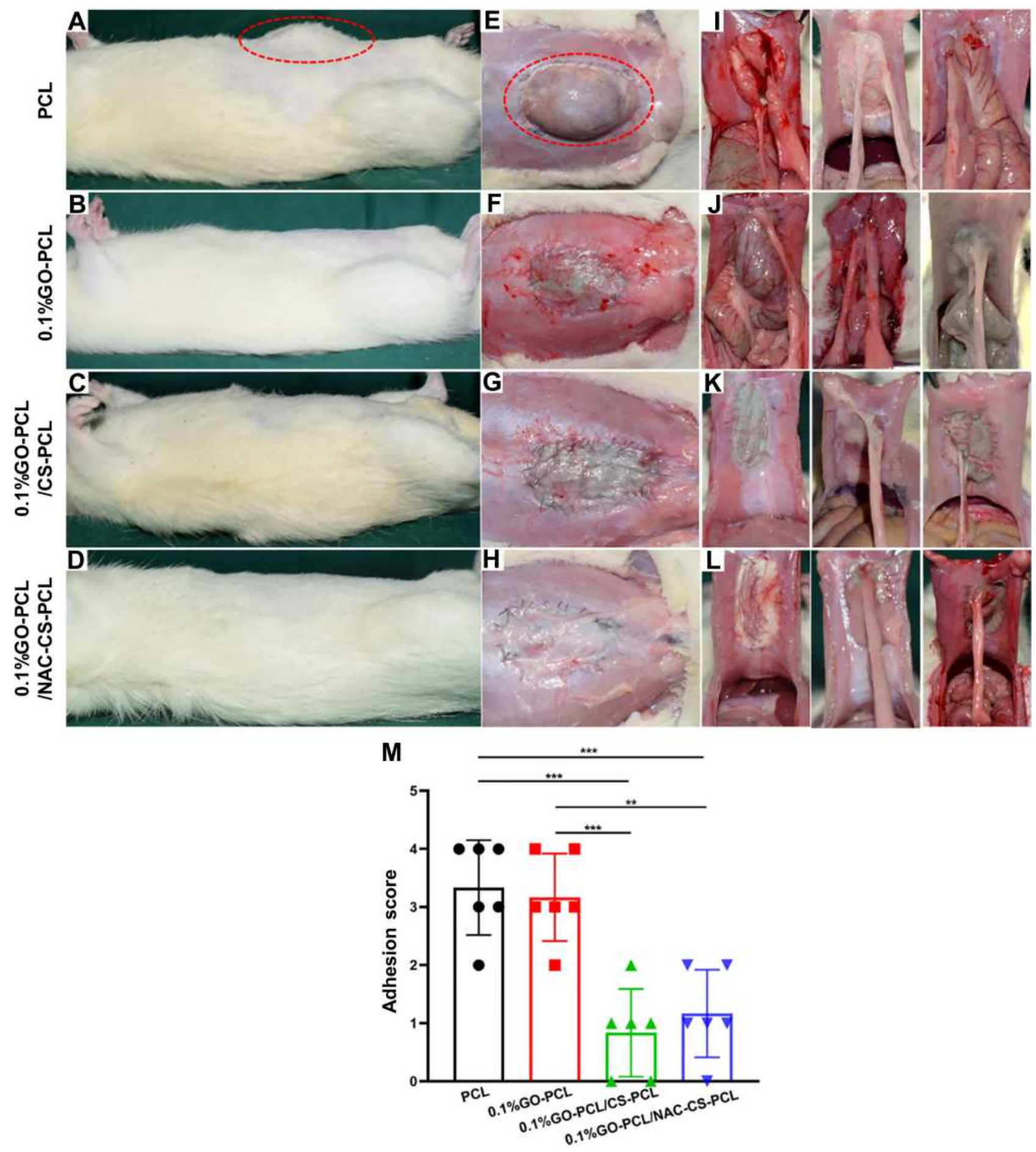

Figure 5 Evaluation of hernia and adhesion formation during the repair of full-thickness abdominal wall defects in rats.

Notes: $(\mathbf{A}-\mathbf{H})$ hernia formation on different scaffolds during the repair of abdominal wall defect. The red dotted round frame shows the formed hernia on PCL scaffolds. (I$\mathbf{L})$ the adhesion formation of different scaffolds during the repair of abdominal wall defect. $(\mathbf{M})$ histogram of adhesion score (mean $\pm \mathrm{SD}$; $* * \mathrm{P}<0.0 \mathrm{I}$, $* * * \mathrm{P}<0.00 \mathrm{I})$.

On the other hand, a large amount of collagen was deposited in the junctional area for all the groups except for the PCL group. In the $0.1 \% \mathrm{GO}-\mathrm{PCL}$ and $0.1 \% \mathrm{GO}-\mathrm{PCL} / \mathrm{CS}-\mathrm{PCL}$ groups, and particularly for the $0.1 \% \mathrm{GO}-\mathrm{PCL} / \mathrm{NAC}-\mathrm{CS}$ PCL group, the collagen fibers were more aligned, and appeared to be more compact and thicker, compared to the thin and loose reticular framework of the PCL group. Through quantitative analysis, we found that compared to other groups, the $0.1 \% \mathrm{GO}-\mathrm{PCL} / \mathrm{NAC}-\mathrm{CS}-\mathrm{PCL}$ group had the most collagen deposition as shown in Figure 7M. The 

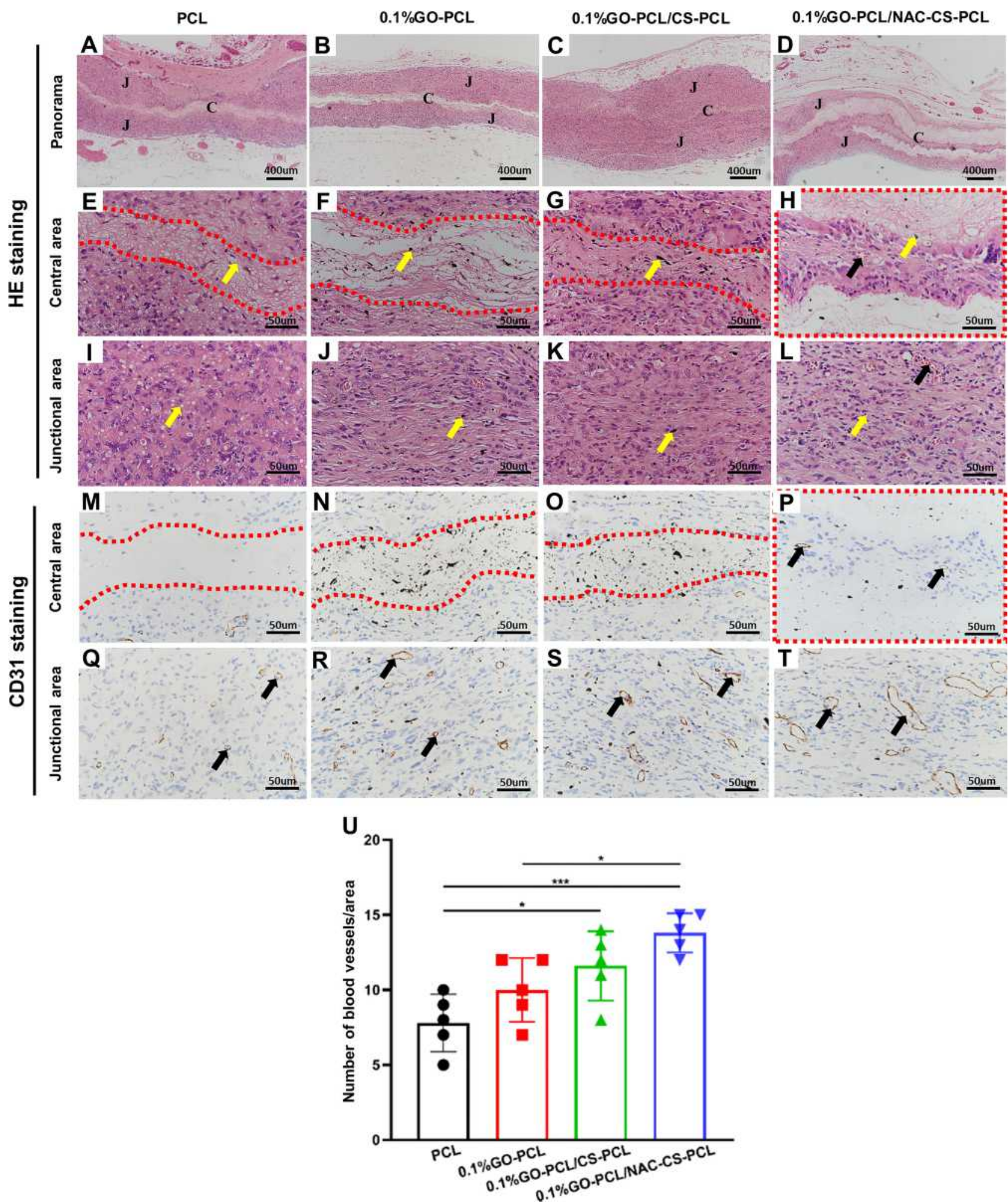

Figure 6 Histological analysis of the scaffolds 2 months post-implantation.

Notes: (A-L) H\&E staining and (M-T) CD3I immunohistochemical staining of explants at 2 months post-implantation. Capital letter $\mathrm{C}$ represents the central area, and capital letter J represents the junctional area. High magnification images of the central area are within the red dotted line. The yellow arrows and black arrows indicate the residual scaffold materials (E and I: PCL; F-H, J-L: GO) and new blood vessels, respectively. (U) histogram of the number of blood vessels/area (mean \pm SD; $*$ P $<0.05$, $* * * \mathrm{P}<0.001)$. 
images of Sirius red staining under a polarized light microscope are shown in Figure 7I-L. From the images, almost all the collagen was seen as red fibers under polarized light, suggesting that the collagen formed was mostly type I collagen rather than other types. $^{26}$

\section{Discussion}

Due to the various limitations of traditional synthetic and biological meshes, there are currently a lot of studies on the development of well-functioning meshes to repair abdominal wall defects. In order to avoid the development of abdominal adhesions normally associated with synthetic meshes, $\mathrm{Hu}$ et al used Mussel-inspired copolymer-coated polypropylene mesh to repair abdominal wall defects and achieved good anti-adhesion effect. ${ }^{27}$ On the other hand, in order to overcome the issue of insufficient mechanical strength associated with biological meshes, Song et al seeded tenocyte on SIS and used it to repair abdominal wall defects. The results showed that this composite mesh had sufficient strength to prevent the formation of hernias. ${ }^{28}$ However, despite its successful use in the repair of abdominal wall defects in rats, it is associated with
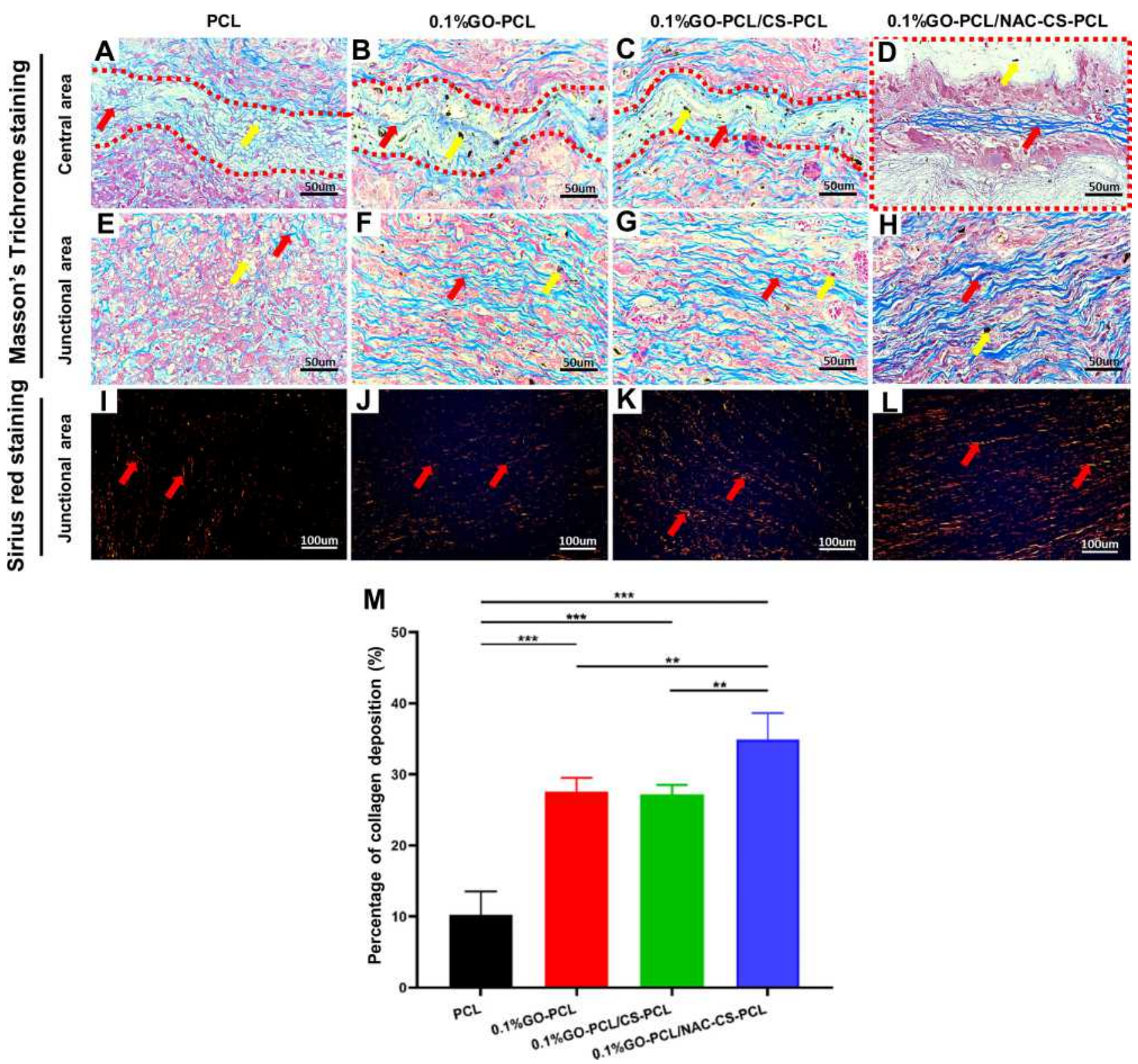

Figure 7 New collagen deposition on the scaffolds 2 months post-implantation.

Notes: (A-H) Masson's Trichrome staining and (I-L) Sirius red staining of explants under polarized light microscope at 2 months post-implantation. The yellow arrows and red arrows indicate the residual scaffold materials (A and E: PCL; B-D, F-H: GO) and new collagen tissues, respectively. (M) histogram of the percentage of collagen deposition per area (mean $\pm \mathrm{SD} ; * * \mathrm{P}<0.01, * * * \mathrm{P}<0.001)$. 
great difficulties in clinical application. In our study, we used electrospinning technology to develop a doublelayered nanofiber scaffold (0.1\%GO-PCL/NAC-CS-PCL) for the reconstruction of abdominal wall defects. The incorporation of GO improved the mechanical strength of the scaffold and avoided the formation of hernia. In addition, the CS integrated in the inner layer successfully prevented the formation of adhesions. Moreover, the NAC loaded in the scaffold played a further role in promoting vascularization and collagen deposition to some extent.

However, it should be noted that although GO played a role in improving the biomechanical strength of the scaffold in our study, its use in tissue engineering is highly controversial due to its cytotoxicity, and this may be the main reason that affects cell proliferation..$^{29,30}$ Thus, in order to obtain a scaffold with good mechanical properties and biocompatibility, we tested the effect of the different concentrations of GO-PCL scaffolds on mechanical strength and cell proliferation rate. The results suggested that compared with high-concentration GO-PCL $(0.5 \%$ GO-PCL, 1.0\%GO-PCL) scaffolds, low-concentration GO-PCL (0.1\%GO-PCL) scaffolds had better cell compatibility, as well as good tensile strength. However, low concentrations of GO still had a slight adverse effect on cell growth compared to simple PCL scaffolds (Figure 2E). To overcome this, an inner scaffold (CS-PCL) was used to cover its surface and NAC was cross-linked to the double-layered scaffold. These measures were able to offset the adverse effects associated with GO as shown in the CCK8 results (Figure 4B), which may be attributed to the blocking effect of the inner scaffold and the growth promoting effects of NAC. In addition, it is worth noting that although GO reduced the cell proliferation rate in in vitro assays, results from in vivo assays showed that addition of GO (0.1\%GO-PCL groups) resulted in the formation of more collagen compared to the simple PCL groups (Figure 7). Therefore, low concentrations of GO may not have significant side effects during application in vivo. Moreover, GO can be degraded in vivo and excreted by multiple organs. ${ }^{31}$ In our study, histological staining showed the presence of GO debris in the central areas as well as the junctional areas since GO particles can be taken up by plasma membrane or macrophages according to their size. ${ }^{32}$ Kurapati et al also reported that GO can be degraded by myeloperoxidase in vivo. ${ }^{33}$ Therefore, it is relatively safe to use low concentration GO composite scaffolds to repair abdominal wall defects.
In addition to providing sufficient mechanical strength and good biocompatibility, reducing postoperative adhesion is another key issue. In current clinical practice, there are two main methods for preventing postoperative abdominal adhesions. One is to improve surgical techniques to minimize bleeding and tissue damage, and the other is to use anti-adhesion biomaterials. ${ }^{34}$ In our study, the electrospinning technology was used to develop the inner film of the double-layer scaffolds using Chitosan and PCL in order to prevent the formation of adhesions. Although pure CS can also be used for electrospinning, the electrospinning of pure CS is problematic due to the high instability of the polymer jet caused by the repulsive forces between protonated amino groups. ${ }^{35}$ Therefore, we mixed PCL with CS to make it stable for electrospinning. There are several mechanisms that have suggested to explain how chitosan prevents the formation of adhesions. First, chitosan has local hemostatic and antibacterial capabilities, which can effectively reduce the inflammatory response around the wound, thereby further preventing the formation of adhesions. ${ }^{36}$ These two properties have the added advantage of promoting wound healing. Second, some studies have suggested that the nanofiber meshes of CS act as biological barriers between exfoliated peritoneum and abdominal organs. ${ }^{37,38}$ Third, a study by Chatelet et al suggested that chitosan inhibits the proliferation of fibroblasts which in turn reduces the formation of collagen fibers involved in the formation of adhesions. ${ }^{39}$ However, in our in vivo assays, there was no decrease in the synthesis of collagen fibers in the $0.1 \% \mathrm{GO}-\mathrm{PCL} / \mathrm{CS}$ PCL group and $0.1 \% \mathrm{GO}-\mathrm{PCL} / \mathrm{NAC}-\mathrm{CS}-\mathrm{PCL}$ group as shown in Figure 7. This may be due to the lower concentrations of chitosan used or the regulating effects of GO and NAC.

In our study, in order to accelerate the repair of abdominal wall defects, NAC was cross-linked with the $0.1 \%$ GO-PCL/CS-PCL scaffold. As a ROS scavenger, NAC regulates excessive inflammatory response and prevents oxidative stress triggered by ROS, and further achieves the purpose of promoting recovery process. ${ }^{40,41}$ Tsai et al demonstrated that NAC could induce collagenous expression of MMP-1 via the PKC/Stat3 signaling pathway. ${ }^{42}$ And Albasanz-Puig et al reported that NAC could activate Stat3 to regulate vascular endothelial growth factor and facilitate the growth of new vessels. ${ }^{43}$ During the twomonth observation period, there was significant deposition of collagen in the $0.1 \% \mathrm{GO}-\mathrm{PCL} / \mathrm{NAC}-\mathrm{CS}-\mathrm{PCL}$ group compared to the $0.1 \% \mathrm{GO}-\mathrm{PCL} / \mathrm{CS}-\mathrm{PCL}$ group (Figure 7) 
and even a small amount of cells and neovascularization were seen in the central area, which was in accordance with the results of previous study. However, there was no significant difference in blood vessel count in the junctional area between the two groups. As to why the promotion effect is not significant, it may be attributed to the following reasons: (1) In our study, the concentration of CS used was low, so the loaded NAC was limited. (2) When cross-linking NAC, the concentration we selected was $0.5 \mathrm{mg} / \mathrm{mL}$, which was based on the concentration we used in a previous study on wound repair. There is a need to determine if the concentration is sufficient for the repair of abdominal wall defects. (3) Although the $0.1 \%$ GO-PCL /NAC-CS-PCL scaffold had a certain sustained release of $\mathrm{NAC}$, the overall release is not good, and according to the drug release curve, the amount of NAC released is low (Figure 3E).

Finally, it should be noted that although the doublelayer patches we prepared achieved the goals of preventing the development of hernia and adhesions, they did not solve the problem of abdominal wall muscle tissue regeneration induction. There is therefore a need for further studies to develop patches that can overcome this problem.

\section{Conclusion}

In summary, we successfully constructed a double-layer scaffold (0.1\%GO-PCL/NAC-CS-PCL) through electrospinning technology. Our findings revealed that the scaffolds had sufficient mechanical strength to prevent the formation of hernia. In addition, the inner scaffolds (CSPCL) played an important role in preventing formation of adhesions. Although cross-linked NAC had limited effectiveness in promoting vascularization, it still had a positive effect in promoting wound repair. The scaffold is easy to prepare and provides a new choice for improving the repair of abdominal wall defects.

\section{Acknowledgments}

This study was funded by the National Key R\&D Program of China (2019YFA0110500), the National Natural Science Foundation of China (No. 8201001114, 81873941), and Frontier Application Project of Wuhan (2020020601012219).

\section{Disclosure}

The authors report no conflicts of interest in this work.

\section{References}

1. Poulose BK, Shelton J, Phillips S, et al. Epidemiology and cost of ventral hernia repair: making the case for hernia research. Hernia. 2012;16(2):179-183. doi:10.1007/s10029-011-0879-9

2. Wen W, Majerus B, Van De Moortel M, et al. Laparoscopic ventral hernia repair using a composite mesh with polypropylene and expanded polytetrafluoroethylene: a prospective, multicentre registry. Acta Chir Belg. 2017;117(5):295-302. doi:10.1080/ 00015458.2017.1313526

3. Ueno T, Pickett LC, de la Fuente SG, Lawson DC, Pappas TN. Clinical application of porcine small intestinal submucosa in the management of infected or potentially contaminated abdominal defects. J Gastrointest Surg. 2004;8(1):109-112. doi:10.1016/j. gassur.2003.09.025

4. Patton JJ, Berry S, Kralovich KA. Use of human acellular dermal matrix in complex and contaminated abdominal wall reconstructions. Am J Surg. 2007;193(3):360-363, 363. doi:10.1016/j. amjsurg.2006.09.021

5. Patel NG, Ratanshi I, Buchel EW. The best of abdominal wall reconstruction. Plast Reconstr Surg. 2018;141(1):113e-136e. doi:10.1097/PRS.0000000000003976

6. Huang HY, Chen LQ, Sun W, et al. Collagenase IV and clusterin-modified polycaprolactone-polyethylene glycol nanoparticles for penetrating dense tumor tissues. Theranostics. 2021;11 (2):906-924. doi:10.7150/thno.47446

7. Liu Z, Zhu X, Tang R. Electrospun scaffold with sustained antibacterial and tissue-matched mechanical properties for potential application as functional mesh. Int $J$ Nanomedicine. 2020;15:4991-5004. doi:10.2147/IJN.S248970

8. Hympanova L, Mori da Cunha MGMC, Rynkevic R, et al. Experimental reconstruction of an abdominal wall defect with electrospun polycaprolactone-ureidopyrimidinone mesh conserves compliance yet may have insufficient strength. $J$ Mech Behav Biomed. 2018;88:431-441. doi:10.1016/j.jmbbm.2018.08.026

9. Chen H, Müller MB, Gilmore KJ, Wallace GG, Li D. Mechanically strong, electrically conductive, and biocompatible graphene paper. Adv Mater. 2008;20(18):3557-3561. doi:10.1002/adma.200800757

10. Song J, Gao H, Zhu G, Cao X, Shi X, Wang Y. The preparation and characterization of polycaprolactone/graphene oxide biocomposite nanofiber scaffolds and their application for directing cell behaviors. Carbon. 2015;95:1039-1050. doi:10.1016/j. carbon.2015.09.011

11. Figueroa T, Aguayo C, Fernandez K. Design and characterization of chitosan-graphene oxide nanocomposites for the delivery of proanthocyanidins. Int $J$ Nanomedicine. 2020;15:1229-1238. doi: 10.2147/IJN.S240305

12. Di Martino A, Sittinger M, Risbud MV. Chitosan: a versatile biopolymer for orthopaedic tissue-engineering. Biomaterials. 2005;26 (30):5983-5990. doi:10.1016/j.biomaterials.2005.03.016

13. Cheng F, Wu Y, Li H, et al. Biodegradable N, O-carboxymethyl chitosan/oxidized regenerated cellulose composite gauze as a barrier for preventing postoperative adhesion. Carbohydr Polym. 2019;207:180-190. doi:10.1016/j.carbpol.2018.10.077

14. Ko JE, Ko YG, Kim WI, Kwon OK, Kwon OH. Nanofiber mats composed of a chitosan-poly(d,1-lactic-co-glycolic acid)-poly(ethylene oxide) blend as a postoperative anti-adhesion agent. $J$ Biomed Mater Res B Appl Biomater. 2017;105(7):1906-1915. doi:10.1002/ jbm.b.33726

15. Hou J, Chen L, Zhou M, et al. Multi-layered polyamide/collagen scaffolds with topical sustained release of $\mathrm{N}$-acetylcysteine for promoting wound healing. Int J Nanomedicine. 2020;15:1349-1361. doi:10.2147/IJN.S232190

16. Zayed MA, Wei X, Park KM, et al. N-Acetylcysteine accelerates amputation stump healing in the setting of diabetes. FASEB J. 2017;31(6):2686-2695. doi:10.1096/fj.201601348R 
17. Suha T, Asli M, Aynur S, et al. Effects of N-acetylcysteine and ethyl pyruvate on ischemia-reperfusion injury in experimental electrical burn model. Am J Emerg Med. 2016;34(7):1217-1224. doi:10.1016/ j.ajem.2016.03.032

18. Li J, Zhou C, Luo C, et al. N-acetyl cysteine-loaded graphene oxide-collagen hybrid membrane for scarless wound healing. Theranostics. 2019;9(20):5839-5853. doi:10.7150/thno.34480

19. Eom S, Park SM, Hong H, et al. Hydrogel-assisted electrospinning for fabrication of a 3D complex tailored nanofiber macrostructure. Acs Appl Mater Inter. 2020;12(46):51212-51224. doi:10.1021/acsami.0c14438

20. Liu S, Mou S, Zhou C, et al. Off-the-shelf biomimetic graphene oxide-collagen hybrid scaffolds wrapped with osteoinductive extracellular matrix for the repair of cranial defects in rats. Acs Appl Mater Inter. 2018;10(49):42948-42958. doi:10.1021/acsami.8b11071

21. Park J, Kim S. Antimicrobial filtration with electrospun poly(vinyl alcohol) nanofibers containing benzyl triethylammonium chloride: immersion, leaching, toxicity, and filtration tests. Chemosphere. 2017;167:469-477. doi:10.1016/j.chemosphere.2016.10.030

22. Wang J, Wu D, Zhang Z, et al. Biomimetically ornamented rapid prototyping fabrication of an apatite-collagen-polycaprolactone composite construct with nano-micro-macro hierarchical structure for large bone defect treatment. ACS Appl Mater Interfaces. 2015;7 (47):26244-26256. doi:10.1021/acsami.5b08534

23. Walker AP, Henderson J, Condon RE. Double-layer prostheses for repair of abdominal wall defects in a rabbit model. J Surg Res. 1993;55(1):32-37. doi:10.1006/jsre.1993.1104

24. Hou J, Chen L, Liu Z, et al. Sustained release of N-acetylcysteine by sandwich structured polycaprolactone/collagen scaffolds for wound healing. J Biomed Mater Res A. 2019;107(7):1414-1424. doi:10.1002/jbm.a.36656

25. Wan C, Chen B. Poly(epsilon-caprolactone)/graphene oxide biocomposites: mechanical properties and bioactivity. Biomed Mater. 2011;6 (5):55010. doi:10.1088/1748-6041/6/5/055010

26. Junqueira LC, Bignolas G, Brentani RR. Picrosirius staining plus polarization microscopy, a specific method for collagen detection in tissue sections. Histochem J. 1979;11(4):447-455. doi:10.1007/BF01002772

27. Hu W, Lu S, Zhang Z, et al. Mussel-inspired copolymer-coated polypropylene mesh with anti-adhesion efficiency for abdominal wall defect repair. Biomater Sci UK. 2019;7:1323-1334. doi:10.1039/C8BM01198B

28. Song Z, Peng Z, Liu Z, Yang J, Tang R, Gu Y. Reconstruction of abdominal wall musculofascial defects with small intestinal submucosa scaffolds seeded with tenocytes in rats. Tissue Eng Part A. 2013;19(13-14):1543-1553. doi:10.1089/ten.tea.2011.0748

29. Fang H, Luo C, Liu S, et al. A biocompatible vascularized graphene oxide (GO)-collagen chamber with osteoinductive and anti-fibrosis effects promotes bone regeneration in vivo. Theranostics. 2020;10 (6):2759-2772. doi:10.7150/thno.42006

30. Kumar S, Chatterjee K. Comprehensive review on the use of graphene-based substrates for regenerative medicine and biomedical devices. ACS Appl Mater Interfaces. 2016;8(40):26431-26457. doi:10.1021/acsami.6b09801

International Journal of Nanomedicine

\section{Publish your work in this journal}

The International Journal of Nanomedicine is an international, peerreviewed journal focusing on the application of nanotechnology in diagnostics, therapeutics, and drug delivery systems throughout the biomedical field. This journal is indexed on PubMed Central, MedLine, CAS, SciSearch ${ }^{\mathbb{R}}$, Current Contents ${ }^{\mathbb{R}} /$ Clinical Medicine, $^{2}$
31. Tonelli FM, Goulart VA, Gomes KN, et al. Graphene-based nanomaterials: biological and medical applications and toxicity. Nanomedicine (Lond). 2015;10(15):2423-2450. doi:10.2217/ nnm. 15.65

32. Ma J, Liu R, Wang X, et al. Crucial role of lateral size for graphene oxide in activating macrophages and stimulating pro-inflammatory responses in cells and animals. Acs Nano. 2015;9(10):10498-10515. doi:10.1021/acsnano.5b04751

33. Kurapati R, Russier J, Squillaci MA, et al. Dispersibility-dependent biodegradation of graphene oxide by myeloperoxidase. Small. 2015;11(32):3985-3994. doi:10.1002/smll.201500038

34. Lin L, Luo J, Yuan F, et al. In situ cross-linking carbodiimide-modified chitosan hydrogel for postoperative adhesion prevention in a rat model. Mater Sci Eng C. 2017;81:380-385. doi:10.1016/j.msec.2017.07.024

35. Geng X, Kwon OH, Jang J. Electrospinning of chitosan dissolved in concentrated acetic acid solution. Biomaterials. 2005;26 (27):5427-5432. doi:10.1016/j.biomaterials.2005.01.066

36. Li L, Wang N, Jin X, et al. Biodegradable and injectable in situ cross-linking chitosan-hyaluronic acid based hydrogels for postoperative adhesion prevention. Biomaterials. 2014;35(12):3903-3917. doi:10.1016/j.biomaterials.2014.01.050

37. Zhu L, Peng L, Zhang YQ. The processing of chitosan and its derivatives and their application for postoperative anti-adhesion. Mini Rev Med Chem. 2015;15(4):330-337. doi:10.2174/ 1389557515666150227110547

38. Xu C, Lei C, Meng L, Wang C, Song Y. Chitosan as a barrier membrane material in periodontal tissue regeneration. $J$ Biomed Mater Res B Appl Biomater. 2012;100B(5):1435-1443. doi:10.1002/jbm.b.32662

39. Chatelet C, Damour O, Domard A. Influence of the degree of acetylation on some biological properties of chitosan films. Biomaterials. 2001;22(3):261-268. doi:10.1016/S0142-9612(00)00183-6

40. Shen Y, Gong S, Li J, et al. Co-loading antioxidant N-acetylcysteine attenuates cytotoxicity of iron oxide nanoparticles in hypoxia/reoxygenation cardiomyocytes. Int $J$ Nanomed. 2019;14:6103-6115. doi:10.2147/IJN.S209820

41. Csontos C, Rezman B, Foldi V, et al. Effect of N-acetylcysteine treatment on oxidative stress and inflammation after severe burn. Burns. 2012;38(3):428-437. doi:10.1016/j.burns.2011.09.011

42. Tsai ML, Huang HP, Hsu JD, et al. Topical N-acetylcysteine accelerates wound healing in vitro and in vivo via the $\mathrm{PKC} / \mathrm{Stat} 3$ pathway. Int J Mol Sci. 2014;15(5):7563-7578. doi:10.3390/ijms15057563

43. Albasanz-Puig A, Murray J, Namekata M, Wijelath ES. Opposing roles of STAT-1 and STAT-3 in regulating vascular endothelial growth factor expression in vascular smooth muscle cells. Biochem Biophys Res Commun. 2012;428(1):179-184. doi:10.1016/j. bbrc.2012.10.037

Journal Citation Reports/Science Edition, EMBase, Scopus and the Elsevier Bibliographic databases. The manuscript management system is completely online and includes a very quick and fair peer-review system, which is all easy to use. Visit http://www.dovepress.com/ testimonials.php to read real quotes from published authors. 